\title{
On the Neumann eigenvalues for second-order Sturm-Liouville difference equations
}

\section{Yan-Hsiou Cheng ${ }^{1 *}$ (])}

"Correspondence:

yhcheng@tea.ntue.edu.tw

'Department of Mathematics and

Information Education, National

Taipei University of Education, Taipei

106, Taiwan

\begin{abstract}
The paper is concerned with the Neumann eigenvalues for second-order Sturm-Liouville difference equations. By analyzing the new discriminant function, we show the interlacing properties between the periodic, antiperiodic, and Neumann eigenvalues. Moreover, when the potential sequence is symmetric and symmetric monotonic, we show the order relation between the first Dirichlet eigenvalue and the second Neumann eigenvalue, and prove that the minimum of the first Neumann eigenvalue gap is attained at the constant potential sequence.
\end{abstract}

MSC: 39A12; 15A42

Keywords: Second-order difference equations; Eigenvalue gap; Neumann eigenvalues

\section{Introduction}

Consider the second-order Sturm-Liouville difference equation

$$
-\nabla\left(\Delta y_{n}\right)+q_{n} y_{n}=\lambda y_{n} \quad \text { on }[0, N-1]
$$

where the potential sequence $q_{i} \geq 0$ for $i=0,1,2, \ldots, N-1, \Delta$ is the forward difference operator $\left(\triangle y_{n}=y_{n+1}-y_{n}\right), \nabla$ is the backward difference operator $\left(\nabla y_{n}=y_{n}-y_{n-1}\right)$, and the bracket $[0, N-1]$ means the integers in $[0, N-1]$. Note that equation (1) can be rewritten as the recurrence formula

$$
y_{n+1}=\left(2+q_{n}-\lambda\right) y_{n}-y_{n-1} \quad \text { on }[0, N-1]
$$

or the matrix formula

$$
(D+Q) \vec{y}=\lambda \vec{y}
$$

(c) The Author(s) 2020. This article is licensed under a Creative Commons Attribution 4.0 International License, which permits use, sharing, adaptation, distribution and reproduction in any medium or format, as long as you give appropriate credit to the original author(s) and the source, provide a link to the Creative Commons licence, and indicate if changes were made. The images or other third party material in this article are included in the article's Creative Commons licence, unless indicated otherwise in a credit line to the material. If material is not included in the article's Creative Commons licence and your intended use is not permitted by statutory regulation or exceeds the permitted use, you will need to obtain permission directly from the copyright holder. To view a copy of this licence, visit http://creativecommons.org/licenses/by/4.0/. 
where $\vec{y}$ is a vector in $\mathbb{R}^{N}, Q$ is a diagonal matrix whose diagonal elements are $q_{0}, q_{1}, \ldots$, $q_{N-1}$, and $D$ is the $N \times N$ tridiagonal matrix of the form

$$
D=\left[\begin{array}{cccccc}
2 & -1 & 0 & \ldots & \ldots & 0 \\
-1 & 2 & -1 & 0 & \ldots & 0 \\
0 & -1 & 2 & \ddots & \ldots & \vdots \\
\vdots & 0 & \ddots & \ddots & \ddots & \vdots \\
\vdots & \vdots & \vdots & \ddots & \ddots & -1 \\
0 & 0 & \cdots & \cdots & -1 & 2
\end{array}\right] .
$$

It is clear that if $u(x) \in C^{2}\left(x_{0}-h, x_{0}+h\right)$ for $h>0$, then there exists $\eta \in\left[x_{0}-h, x_{0}+h\right]$ such that

$$
u\left(x_{0}-h\right)-2 u\left(x_{0}\right)+u\left(x_{0}+h\right)=u^{\prime \prime}(\eta) h^{2} .
$$

Equation (1) can be regarded as a discrete analogue of the Sturm-Liouville problem

$$
-u^{\prime \prime}(x)+q(x) u(x)=\lambda u(x) \quad \text { on }(0,1)
$$

Sturm-Liouville problem (2) has been widely studied. For the potential function $q \in L^{1}$, the eigenvalues of Sturm-Liouville problem (2) with separated boundary conditions are real, simple, increasing and tend to infinity [3, 21, 23]. By defining Hill's discriminant of (2) by

$$
H(\lambda)=y_{1}(1, \lambda)+y_{2}^{\prime}(1, \lambda)
$$

where $y_{1}(x, \lambda)$ and $y_{2}(2, \lambda)$ are solutions of (2) and satisfy

$$
y_{1}(0, \lambda)=y_{2}^{\prime}(0, \lambda)=1, \quad y_{1}^{\prime}(0, \lambda)=y_{2}(0, \lambda)=0,
$$

one can show that $[3,9,22]$ the periodic eigenvalues $\left\{\lambda_{n}\right\}_{n \geq 0}$, anti-periodic eigenvalues $\left\{\lambda_{n}^{\prime}\right\}_{n \geq 1}$, Dirichlet eigenvalues $\left\{\mu_{n}\right\}_{n \geq 1}$, and Neumann eigenvalues $\left\{v_{n}\right\}_{n \geq 0}$ satisfy $H\left(\lambda_{n}\right)=$ 2, $H\left(\lambda_{n}^{\prime}\right)=-2, H\left(\lambda_{n}^{\prime}\right)=-2, H\left(\mu_{2 n+1}\right)<-2, H\left(\mu_{2 n}\right)>2, H\left(v_{2 n}\right)>2$, and $H\left(v_{2 n+1}\right)<-2$. In particular, we also know $v_{0} \leq \lambda_{0}$ and

$$
\cdots \leq \lambda_{2 n-2}<\lambda_{2 n-1}^{\prime} \leq{ }^{\nu_{2 n-1}} \leq \lambda_{2 n}^{\prime}<\lambda_{2 n-1} \leq{ }_{\mu_{2 n}}^{\nu_{2 n}} \leq \lambda_{2 n}<\lambda_{2 n+1}^{\prime} \leq \cdots .
$$

The periodic Sturm-Liouville problem is also called Hill's equation. The intervals $\left(\lambda_{2 n-1}^{\prime}, \lambda_{2 n}^{\prime}\right)$ and $\left(\lambda_{2 n-1}, \lambda_{2 n}\right)$ are called the $(2 n-1)$ th and $2 n$th instability intervals. The interval $\left(-\infty, \lambda_{0}\right)$ is called the zero-th instability interval. The name instability interval is used because, for all $\lambda$ in these intervals, all nontrivial solutions of (2) are unbounded in $(-\infty, \infty)$. In 1946, Borg showed [5] that, for Hill's equation, the potential $q$ is constant if and only if all instability intervals, except the zero-th, are absent. He also showed that all odd instability intervals $\left(\lambda_{2 n-1}^{\prime}, \lambda_{2 n}^{\prime}\right)$ vanish if and only if $q$ has period $1 / 2$. Later on, 
Hochstadt [14] generalized Borg's results to show that if $q$ is $C^{1}$, then $q$ has period $1 / n$ if and only if all those finite instability intervals whose index is not a multiple of $n$ vanish. In particular, Hochstadt also showed that [11] if all but except one instability interval vanish, then the potential function has to be an elliptic function. Furthermore, it was proved that the first instability interval is absent if and only if the potential function is constant when the potential function $q$ is assumed to be symmetric single-well [16]or single-well [6].

Recently, there have been a number of studies on minimum Dirichlet eigenvalue gaps of the Sturm-Liouville equations (2) with convex potentials [19, 20], symmetric singlewell potentials [1], or single-well potentials [15], while the symmetric 1-step function is the potential function in $E[h, H, M] \equiv\left\{q \in \mathrm{PC}(0,1): h \leq q \leq H\right.$ a.e. and $\left.\int_{0}^{1} q=M\right\}$ giving the minimal Dirichlet eigenvalue gap [8]. Later on, Cheng et al. [6, 7] showed that if the potential function $q$ is single-well with transition point $a=1 / 2$, then $v_{1} \geq \mu_{1}$ and $v_{1}-v_{0} \geq \pi^{2}$. Equality holds if and only if $q$ is constant.

In this paper, we study the second-order difference equations (1). So far, there have been results on the second-order difference equations (1) which are analogue to the continuous Sturm-Liouville equation (2). Using the information on more than one set of eigenvalues, the potential sequence can be determined uniquely, for example, two sets of eigenvalues $[12,13]$, one set of eigenvalues plus a symmetric potential sequence [10], and one set of eigenvalues plus partial information of the potential sequence [25].

In 1990, Ashbaugh and Benguria [2] studied the comparison of the eigenvalues of two discrete Sturm-Liouville equations whose potential sequences satisfy certain relation. We say the sequence $\left\{x_{k}\right\}_{k=0}^{N-1}$ is symmetric if $x_{k}=x_{N-1-k}$ for $k=0,1,2, \ldots, N-1$, and the sequence $\left\{x_{k}\right\}_{k=0}^{N-1}$ is quasi-symmetric increasing if $x_{0} \geq x_{N-1} \geq x_{1} \geq \cdots \geq x_{\left[\frac{N}{2}\right]}$. In particular, the sequence $\left\{x_{k}\right\}_{k=0}^{N-1}$ is said to be symmetric increasing if it is symmetric and quasisymmetric increasing. Ashbaugh and Benguria showed that if $\left\{q_{k}\right\}_{k=0}^{N-1}$ is symmetric increasing in (1), then the eigenvalue $\left\{\mu_{k}\right\}_{k=1}^{N-1}$ satisfies

$$
\mu_{2}-\mu_{1} \geq 2\left[\cos \left(\frac{\pi}{N}\right)-\cos \left(\frac{2 \pi}{N}\right)\right]
$$

Equality holds if and only if $q_{k}=q_{0}$ for $k=0,1,2, \ldots, N-1$. Note that if $q_{k}=0$ for $k=$ $0,1,2, \ldots, N-1$, then

$$
\mu_{k}=4 \sin ^{2}\left(\frac{k \pi}{2 N}\right)=2\left[1-\cos \left(\frac{k \pi}{N}\right)\right], \quad k=1,2, \ldots, N-1 .
$$

Furthermore, the system of (1) with other self-adjoint boundary conditions has also been investigated [3, 18]. Jirari in 1995 showed that problem (1) with the boundary conditions

$$
y_{-1}-\alpha y_{0}=y_{N}-\beta y_{N-1}=0,
$$

where $\alpha, \beta \in \mathbb{R}$ has $N$ real and simple eigenvalues. And recently, Ji and Yang [17] studied the eigenvalue comparison of (1) and (4). In particular, they also showed that if $q_{i}=0$ for $i=0,1,2, \ldots, N-1$, then the first eigenvalue is simple and associated with the vector whose entries are of all ones. 
In 2005, Wang and Shi [26] (see also [24]) were concerned with the eigenvalues for (1) coupled with the periodic boundary conditions

$$
y_{-1}=y_{N-1}, \quad y_{0}=y_{N}
$$

the antiperiodic boundary conditions

$$
y_{-1}=-y_{N-1}, \quad y_{0}=-y_{N},
$$

and the Dirichlet boundary conditions

$$
y_{0}=y_{N}=0
$$

Define the discriminant of (1) by

$$
d(\lambda)=\varphi_{N-1}(\lambda)+\psi_{N}(\lambda)
$$

where $\varphi_{n}$ and $\psi_{n}$ are solutions of (1) satisfying the initial conditions

$$
\varphi_{-1}=\psi_{0}=1, \quad \varphi_{0}=\psi_{-1}=0 .
$$

By the similar argument as the differential equations (see [3, 22]), Wang and Shi showed that the periodic problem (1), (5) and the antiperiodic problem (1), (6) have exactly $N$ real eigenvalues, while the Dirichlet problem (1), (7) has exactly $N-1$ real eigenvalues. Furthermore, they denoted by $\left\{\lambda_{k}\right\}_{k=0}^{N-1},\left\{\tilde{\lambda}_{k}\right\}_{k=1}^{N}$, and $\left\{\mu_{k}\right\}_{k=1}^{N-1}$ the periodic, antiperiodic, and Dirichlet eigenvalues, respectively, and arranged them in the nondecreasing order $\lambda_{0} \leq \lambda_{1} \leq \cdots \leq \lambda_{N-1}, \tilde{\lambda}_{1} \leq \tilde{\lambda}_{2} \leq \cdots \leq \tilde{\lambda}_{N}$, and $\mu_{1}<\mu_{1}<\cdots<\mu_{N-1}$. They showed that a set of these three eigenvalues satisfy the following interlacing properties: if $N$ is odd,

$$
\lambda_{0}<\tilde{\lambda}_{1} \leq \mu_{1} \leq \tilde{\lambda}_{2}<\lambda_{1} \leq \mu_{2} \leq \lambda_{2}<\cdots<\lambda_{N-2} \leq \mu_{N-1} \leq \lambda_{N-1}<\tilde{\lambda}_{N}
$$

and if $N$ is even,

$$
\lambda_{0}<\tilde{\lambda}_{1} \leq \mu_{1} \leq \tilde{\lambda}_{2}<\lambda_{1} \leq \mu_{2} \leq \lambda_{2}<\cdots<\tilde{\lambda}_{N-1} \leq \mu_{N-1} \leq \tilde{\lambda}_{N}<\lambda_{N-1} .
$$

In this paper, we consider the second-order difference equations (1) coupled with the Neumann boundary conditions

$$
y_{-1}-y_{0}=y_{N}-y_{N-1}=0 .
$$

Denote by $\left\{v_{k}\right\}_{k=0}^{N-1}$ the Neumann eigenvalues of the second-order difference equations (1). It is known that if $q_{k}=0$ for $k=0,1,2, \ldots, N-1$, then

$$
v_{k}=4 \sin ^{2}\left(\frac{k \pi}{2 N}\right), \quad k=0,1,2, \ldots, N-1 .
$$

Combined with the result of [26], we will show the interlacing properties of the eigenvalues, which is a discrete analogue result for the continuous Sturm-Liouville problem (see 
$[9,22])$. We shall remark that, in the continuous case, we analyze Hill's discriminant $H(\lambda)$ to obtain the interlacing property (3). But in the discrete case, we need to define another discriminant

$$
f(\lambda)=\varphi_{N-1}(\lambda)+\psi_{N}(\lambda)-\psi_{N-1}(\lambda)
$$

where $\varphi_{n}$ and $\psi_{n}$ are solutions of (1) satisfying the initial conditions

$$
\varphi_{-1}=\psi_{0}=\varphi_{0}=1, \quad \psi_{-1}=0,
$$

to show the interlacing property for the Neumann eigenvalues in Theorem 1. By analyzing this new discriminant $f(\lambda)$, we can prove Theorem 1 .

Theorem 1 Consider the second-order difference equations (1). The eigenvalues satisfy the following interlacing inequality: if $N$ is odd,

$$
v_{0} \leq \lambda_{0}<\tilde{\lambda}_{1} \leq{ }_{v_{1}}^{\mu_{1}} \leq \tilde{\lambda}_{2}<\lambda_{1} \leq{ }_{v_{2}}^{\mu_{2}} \leq \lambda_{2}<\cdots<\lambda_{N-2} \leq{ }_{v_{N-1}}^{\mu_{N-1}} \leq \lambda_{N-1}<\tilde{\lambda}_{N}
$$

and if $N$ is even,

$$
v_{0} \leq \lambda_{0}<\tilde{\lambda}_{1} \leq{ }_{v_{1}}^{\mu_{1}} \leq \tilde{\lambda}_{2}<\lambda_{1} \leq{ }_{v_{2}}^{\mu_{2}} \leq \lambda_{2}<\cdots<\tilde{\lambda}_{N-1} \leq \begin{aligned}
& \mu_{N-1} \\
& v_{N-1}
\end{aligned} \leq \tilde{\lambda}_{N}<\lambda_{N-1}
$$

After obtaining Theorem 1, we will consider the order relation of the first Dirichlet eigenvalue $\mu_{1}$ and the second Neumann eigenvalue $v_{1}$, and the first Neumann eigenvalue gap $v_{1}-v_{0}$. Theorems 2 and 3 can be regarded as discrete analogue results of [6] and [7] respectively for the continuous Sturm-Liouville problem (2).

Theorem 2 Consider the second-order difference equation (1). If $q_{k}$ is symmetric and symmetric decreasing and satisfies $\max _{k \in[0, N-1]} q_{k} \leq v_{1}$, then $\mu_{1} \leq v_{1}$ and the equality holds if and only if $q_{k}=q_{0}$ for all $k \in[0, N-1]$.

Theorem 3 Consider the second-order difference equation (1). If $q_{n}$ is symmetric and symmetric increasing, then $v_{1}-v_{0} \geq 2\left[1-\cos \left(\frac{\pi}{N+1}\right)\right]$ and the equality holds if and only if $q_{k}=q_{0}$ for all $k \in[0, N-1]$.

The paper is organized as follows. Section 2 gives lemmas about the Wronskian and a variation of constant formula which is used in Sect. 3. In Sect. 3, we study the interlacing properties for the periodic, antiperiodic, and Neumann eigenvalues, and use an argument similar to that in $[9,22]$ to prove Theorem 1 . Finally, the proof of Theorem 2 is given in Sect. 4, while the proof of Theorem 3 is given in Sect. 5.

\section{Preliminaries}

In this section, we derive some discrete analogous lemmas of the continuous case. One can refer to [4]. Lemmas 1 and 2 have been shown in [18] (see also [26]) by using a similar argument as the continuous case, so we omit the proofs here. 
Lemma 1 ([18, Theorem 2.2.3]) Let $y$ and $z$ be solutions of

$$
-\nabla\left(\Delta y_{n}\right)+q_{n} y_{n}=\lambda y_{n}, \quad n \in[0, N-1]
$$

and

$$
-\nabla\left(\triangle z_{n}\right)+q_{n} z_{n}=\mu z_{n}, \quad n \in[0, N-1]
$$

respectively. Then, for $0 \leq n \leq N-1$,

$$
(\lambda-\mu) \sum_{j=0}^{n} y_{j} z_{j}=\left(y_{n} z_{n+1}-y_{n+1} z_{n}\right)-\left(y_{-1} z_{0}-y_{0} z_{-1}\right)
$$

Let $\lambda=\mu$ in Lemma 1, we have the following Wronskian-type identity.

Lemma 2 ([18, Theorem 2.2.8]) Let $y$ and $z$ be solutions of (1). Then the Wronskian

$$
W[y, z](n) \equiv\left|\begin{array}{cc}
y_{n+1} & z_{n+1} \\
y_{n+1}-y_{n} & z_{n+1}-z_{n}
\end{array}\right|=y_{n} z_{n+1}-y_{n+1} z_{n}
$$

is a constant on $[-1, N-1]$.

Now, let $\varphi_{n}$ and $\psi_{n}$ be two solutions of (1) satisfying the initial conditions

$$
\varphi_{-1}=\psi_{0}=\varphi_{0}=1, \quad \psi_{-1}=0 .
$$

Note that $\Delta \varphi_{-1}=\varphi_{0}-\varphi_{-1}=0$ and $\Delta \psi_{-1}=\psi_{0}-\psi_{-1}=1$. In particular, we find that, by Theorem 2,

$$
W\left[\varphi_{n}, \psi_{n}\right](N-1)=\varphi_{N-1} \psi_{N}-\varphi_{N} \psi_{N-1}=\varphi_{-1} \psi_{0}-\varphi_{0} \psi_{-1}=1
$$

and it is known that $\varphi_{n}, \psi_{n}$ are two linear independent solutions of (1). The following theorem is similar to [26, Theorem 2.3], but the initial conditions are different.

Theorem 4 For any $\left\{f_{n}\right\}_{n=0}^{N-1} \subseteq \mathbb{R}$ and for any $c_{0}, c_{1} \in \mathbb{R}$, the initial value problem

$$
\begin{aligned}
& -\nabla\left(\triangle z_{n}\right)+\left(q_{n}-\lambda\right) z_{n}=f_{n}, \quad n \in[0, N-1] \\
& z_{-1}=c_{-1}, \quad z_{0}=c_{0}
\end{aligned}
$$

has a unique solution $z$, which can be expressed as

$$
z_{n}=c_{-1} \varphi_{n}+\left(c_{0}-c_{-1}\right) \psi_{n}+\sum_{j=0}^{n-1}\left(\varphi_{n} \psi_{j}-\varphi_{j} \psi_{n}\right) f_{j}, \quad n \in[-1, N]
$$

where $\sum_{j=0}^{-1} \bullet \equiv 0$ 
Proof The technique of the proof is based on the variation of parameters on the differential equation. Let

$$
z_{n}=A_{n} \varphi_{n}+B_{n} \psi_{n}, \quad n \in[-1, N]
$$

be a solution of (11). Then

$$
\Delta z_{n}=A_{n} \Delta \varphi_{n}+\varphi_{n+1} \Delta A_{n}+B_{n} \Delta \psi_{n}+\psi_{n+1} \Delta B_{n}, \quad n \in[-1, N-1] .
$$

Setting

$$
\varphi_{n+1} \Delta A_{n}+\psi_{n+1} \Delta B_{n}=0, \quad n \in[-1, N-1],
$$

we have

$$
\Delta z_{n}=A_{n} \Delta \varphi_{n}+B_{n} \Delta \psi_{n}, \quad n \in[-1, N-1] .
$$

Since $\varphi_{n}$ and $\psi_{n}$ are two solutions of (1), we find

$$
\begin{array}{ll}
\varphi_{n+1}=\left(2+q_{n}-\lambda\right) \varphi_{n}-\varphi_{n-1}, & n \in[0, N-1], \\
\psi_{n+1}=\left(2+q_{n}-\lambda\right) \psi_{n}-\psi_{n-1}, & n \in[0, N-1],
\end{array}
$$

and hence

$$
\begin{aligned}
-\nabla\left(\Delta z_{n+1}\right)= & -A_{n+1} \varphi_{n+2}+A_{n} \varphi_{n+1}+A_{n+1} \varphi_{n+1}-A_{n} \varphi_{n} \\
& -B_{n+1} \psi_{n+2}+B_{n} \psi_{n+1}+B_{n+1} \psi_{n+1}-B_{n} \psi_{n} \\
= & -A_{n+1}\left(\left(2+q_{n+1}-\lambda\right) \varphi_{n+1}-\varphi_{n}\right)+A_{n} \varphi_{n+1}+A_{n+1} \varphi_{n+1}-A_{n} \varphi_{n} \\
& -B_{n+1}\left(\left(2+q_{n+1}-\lambda\right) \psi_{n+1}-\psi_{n}\right)+B_{n} \psi_{n+1}+B_{n+1} \psi_{n+1}-B_{n} \psi_{n} \\
= & -\left(q_{n+1}-\lambda\right)\left(A_{n+1} \varphi_{n+1}+B_{n+1} \psi_{n+1}\right) \\
& -\left(A_{n+1}-A_{n}\right)\left(\varphi_{n+1}-\varphi_{n}\right)-\left(B_{n+1}-B_{n}\right)\left(\psi_{n+1}-\psi_{n}\right) \\
= & -\left(q_{n+1}-\lambda\right) z_{n+1}-\Delta A_{n} \Delta \varphi_{n}-\Delta B_{n} \Delta \psi_{n}
\end{aligned}
$$

for $n \in[-1, N-2]$. Combined with (11), we find

$$
\Delta \varphi_{n} \Delta A_{n}+\Delta \psi_{n} \Delta B_{n}=-f_{n+1}, \quad n \in[-1, N-2] .
$$

Now, solving system (13) and (14) for $\left(\Delta A_{n}, \Delta B_{n}\right)$, we find

$$
\Delta A_{n}=\frac{\psi_{n+1} f_{n+1}}{\varphi_{n+1} \Delta \psi_{n}-\psi_{n+1} \Delta \varphi_{n}}, \quad \Delta B_{n}=\frac{\varphi_{n+1} f_{n+1}}{\psi_{n+1} \Delta \varphi_{n}-\varphi_{n+1} \Delta \psi_{n}}, \quad n \in[-1, N-2] .
$$

By (10), we find $\varphi_{n+1} \Delta \psi_{n}-\psi_{n+1} \Delta \varphi_{n}=1$. Hence

$$
\Delta A_{n}=A_{n+1}-A_{n}=\psi_{n+1} f_{n+1}, \quad \Delta B_{n}=B_{n+1}-B_{n}=-\varphi_{n+1} f_{n+1}, \quad n \in[-1, N-2]
$$


and then, by defining $\sum_{j=0}^{-1} \bullet=0$, we have

$$
A_{n}=A_{-1}+\sum_{j=0}^{n} \psi_{j} f_{j}, \quad B_{n}=B_{-1}-\sum_{j=0}^{n} \varphi_{j} f_{j}, \quad n \in[-1, N-1]
$$

This implies that

$$
z_{n}=A_{n} \varphi_{n}+B_{n} \psi_{n}=A_{-1} \varphi_{n}+B_{-1} \psi_{n}+\sum_{j=0}^{n-1}\left(\varphi_{n} \psi_{j}-\varphi_{j} \psi_{n}\right) f_{j}, \quad n \in[-1, N-1]
$$

By (9) and (12), we find

$$
A_{-1}=c_{-1}, \quad B_{-1}=c_{0}-c_{-1} .
$$

Finally, for $n=N$, we evaluate

$$
\begin{aligned}
z_{N}= & -f_{N-1}+\left(2+q_{N-1}-\lambda\right) z_{N-1}-z_{N-2} \\
= & -f_{N-1}+\left(2+q_{N-1}-\lambda\right)\left[c_{-1} \varphi_{N-1}+\left(c_{0}-c_{-1}\right) \psi_{N-1}+\sum_{j=0}^{N-2}\left(\varphi_{N-1} \psi_{j}-\varphi_{j} \psi_{N-1}\right) f_{j}\right] \\
& -\left[c_{-1} \varphi_{N-2}+\left(c_{0}-c_{-1}\right) \psi_{N-2}+\sum_{j=0}^{N-3}\left(\varphi_{N-2} \psi_{j}-\varphi_{j} \psi_{N-2}\right) f_{j}\right] \\
= & -f_{N-1}+c_{-1}\left[\left(2+q_{N-1}-\lambda\right) \varphi_{N-1}-\varphi_{N-2}\right] \\
& +\left(c_{0}-c_{-1}\right)\left[\left(2+q_{N-1}-\lambda\right) \psi_{N-1}-\psi_{N-2}\right] \\
& +\sum_{j=0}^{N-2}\left[\left(2+q_{N-1}-\lambda\right)\left(\varphi_{N-1} \psi_{j}-\varphi_{j} \psi_{N-1}\right)-\left(\varphi_{N-2} \psi_{j}-\varphi_{j} \psi_{N-2}\right)\right] f_{j} \\
= & -f_{N-1}+c_{-1} \varphi_{N}+\left(c_{0}-c_{-1}\right) \psi_{N}+\sum_{j=0}^{N-2}\left[\varphi_{N} \psi_{j}-\varphi_{j} \psi_{N}\right] f_{j} \\
= & c_{-1} \varphi_{N}+\left(c_{0}-c_{-1}\right) \psi_{N}+\sum_{j=0}^{N-1}\left[\varphi_{N} \psi_{j}-\varphi_{j} \psi_{N}\right] f_{j} .
\end{aligned}
$$

This proof is complete.

\section{Interlacing properties of eigenvalues}

Recall $\varphi_{n}$ and $\psi_{n}$ defined in Sect. 2. It is clear that $\lambda$ is an eigenvalue of (1) and (5) if and only if $c_{1} \varphi_{n}(\lambda)+c_{2} \psi_{n}(\lambda)$ satisfies the periodic boundary conditions, i.e.,

$$
\begin{aligned}
& c_{1} \varphi_{-1}(\lambda)+c_{2} \psi_{-1}(\lambda)=c_{1} \varphi_{N-1}(\lambda)+c_{2} \psi_{N-1}(\lambda), \\
& c_{1} \varphi_{0}(\lambda)+c_{2} \psi_{0}(\lambda)=c_{1} \varphi_{N}(\lambda)+c_{2} \psi_{N}(\lambda) .
\end{aligned}
$$


By (9), we find

$$
\begin{gathered}
c_{1}\left[\varphi_{N-1}(\lambda)-1\right]+c_{2} \psi_{N-1}(\lambda)=0, \\
c_{1}\left[\varphi_{N}(\lambda)-1\right]+c_{2}\left[\psi_{N}(\lambda)-1\right]=0 .
\end{gathered}
$$

The above system has a nontrivial solution $\left(c_{1}, c_{2}\right)$ if and only if

$$
\left|\begin{array}{cc}
\varphi_{N-1}(\lambda)-1 & \psi_{N-1}(\lambda) \\
\varphi_{N}(\lambda)-1 & \psi_{N}(\lambda)-1
\end{array}\right|=0
$$

which implies that, combined with (10),

$$
f(\lambda) \equiv \varphi_{N-1}(\lambda)+\psi_{N}(\lambda)-\psi_{N-1}(\lambda)=2
$$

Hence, we find that $f(\lambda)=2$ if and only if $\lambda$ is a periodic eigenvalue. Similarly, it can be showed that $f(\lambda)=-2$ if and only if $\lambda$ is an antiperiodic eigenvalue. In particular, we have the following lemma.

Lemma $3 f^{\prime}(\lambda)=0$ whenever $f(\lambda)=2$ if and only if $\lambda$ is a multiple eigenvalue of (1) and (5), while $f^{\prime}(\lambda)=0$ whenever $f(\lambda)=-2$ if and only if $\lambda$ is a multiple eigenvalue of (1) and (6).

\section{Proof}

First, we differentiate

$$
-\nabla\left(\Delta \varphi_{n}\right)+q_{n} \varphi_{n}=\lambda \varphi_{n}, \quad-\nabla\left(\Delta \psi_{n}\right)+q_{n} \psi_{n}=\lambda \psi_{n}
$$

with respect to $\lambda$ to obtain

$$
\begin{aligned}
& -\nabla\left(\Delta \varphi_{n}^{\prime}\right)+\left(q_{n}-\lambda\right) \varphi_{n}^{\prime}(\lambda)=\varphi_{n}(\lambda), \\
& -\nabla\left(\Delta \psi_{n}^{\prime}\right)+\left(q_{n}-\lambda\right) \psi_{n}^{\prime}(\lambda)=\psi_{n}(\lambda) .
\end{aligned}
$$

In particular, by (9), we also have

$$
\varphi_{-1}^{\prime}=\psi_{-1}^{\prime}=\varphi_{0}^{\prime}=\psi_{0}^{\prime}=0
$$

By Theorem 4, we obtain

$$
\begin{aligned}
\varphi_{n}^{\prime} & =\sum_{j=0}^{n-1}\left(\varphi_{n}(\lambda) \psi_{j}(\lambda)-\varphi_{j}(\lambda) \psi_{n}(\lambda)\right) \varphi_{j}(\lambda), \quad n \in[-1, N], \\
\psi_{n}^{\prime} & =\sum_{j=0}^{n-1}\left(\varphi_{n}(\lambda) \psi_{j}(\lambda)-\varphi_{j}(\lambda) \psi_{n}(\lambda)\right) \psi_{j}(\lambda), \quad n \in[-1, N] .
\end{aligned}
$$


Hence, we have

$$
\begin{aligned}
f^{\prime}(\lambda)= & \varphi_{N-1}^{\prime}(\lambda)+\psi_{N}^{\prime}(\lambda)-\psi_{N-1}^{\prime}(\lambda) \\
= & \sum_{j=0}^{N-2}\left(\varphi_{N-1}(\lambda) \psi_{j}(\lambda)-\varphi_{j}(\lambda) \psi_{N-1}(\lambda)\right) \varphi_{j}(\lambda) \\
& +\sum_{j=0}^{N-1}\left(\varphi_{N}(\lambda) \psi_{j}(\lambda)-\varphi_{j}(\lambda) \psi_{N}(\lambda)\right) \psi_{j}(\lambda) \\
& -\sum_{j=0}^{N-2}\left(\varphi_{N-1}(\lambda) \psi_{j}(\lambda)-\varphi_{j}(\lambda) \psi_{N-1}(\lambda)\right) \psi_{j}(\lambda) \\
= & \sum_{j=0}^{N-1}\left(\varphi_{N-1}(\lambda) \psi_{j}(\lambda)-\varphi_{j}(\lambda) \psi_{N-1}(\lambda)\right) \varphi_{j}(\lambda) \\
& +\sum_{j=0}^{N-1}\left(\varphi_{N}(\lambda) \psi_{j}(\lambda)-\varphi_{j}(\lambda) \psi_{N}(\lambda)\right) \psi_{j}(\lambda) \\
& -\sum_{j=0}^{N-1}\left(\varphi_{N-1}(\lambda) \psi_{j}(\lambda)-\varphi_{j}(\lambda) \psi_{N-1}(\lambda)\right) \psi_{j}(\lambda) \\
= & -\sum_{j=0}^{N-1}\left\{\left[\varphi_{N-1}(\lambda)-\varphi_{N}(\lambda)\right] \psi_{j}^{2}(\lambda)\right. \\
& \left.+\left[\psi_{N}(\lambda)-\psi_{N-1}(\lambda)-\varphi_{N-1}(\lambda)\right] \varphi_{j}(\lambda) \psi_{j}(\lambda)+\psi_{N-1}(\lambda) \varphi_{j}^{2}(\lambda)\right\} .
\end{aligned}
$$

Denote

$$
I(\lambda) \equiv\left[\begin{array}{cc}
\varphi_{N-1}(\lambda)-\varphi_{N}(\lambda) & \frac{\psi_{N}(\lambda)-\psi_{N-1}(\lambda)-\varphi_{N-1}(\lambda)}{2} \\
\frac{\psi_{N}(\lambda)-\psi_{N-1}(\lambda)-\varphi_{N-1}(\lambda)}{2} & \psi_{N-1}(\lambda)
\end{array}\right], \quad \vec{\omega}=\left[\begin{array}{c}
\psi_{j}(\lambda) \\
\varphi_{j}(\lambda)
\end{array}\right]
$$

and

$$
\begin{aligned}
\delta_{j}(\lambda) \equiv & \vec{\omega}^{T} I \vec{\omega} \\
= & {\left[\varphi_{N-1}(\lambda)-\varphi_{N}(\lambda)\right] \psi_{j}^{2}(\lambda)+\left[\psi_{N}(\lambda)-\psi_{N-1}(\lambda)-\varphi_{N-1}(\lambda)\right] \varphi_{j}(\lambda) \psi_{j}(\lambda) } \\
& +\psi_{N-1}(\lambda) \varphi_{j}^{2}(\lambda) .
\end{aligned}
$$

By (10) and (15), we find

$$
\begin{aligned}
\operatorname{det} I= & \psi_{N-1}(\lambda)\left[\varphi_{N-1}(\lambda)-\varphi_{N}(\lambda)\right]-\left(\frac{\psi_{N}(\lambda)-\psi_{N-1}(\lambda)-\varphi_{N-1}(\lambda)}{2}\right)^{2} \\
= & \psi_{N-1}(\lambda)\left[\varphi_{N-1}(\lambda)-\varphi_{N}(\lambda)\right]-\left(\frac{\psi_{N}(\lambda)-\psi_{N-1}(\lambda)+\varphi_{N-1}(\lambda)}{2}\right)^{2} \\
& +\left[\psi_{N}(\lambda)-\psi_{N-1}(\lambda)\right] \varphi_{N-1}(\lambda) \\
= & \varphi_{N-1}(\lambda) \psi_{N}(\lambda)-\varphi_{N}(\lambda) \psi_{N-1}(\lambda)-1 \\
= & 0 .
\end{aligned}
$$


Hence, the matrix $I(\lambda)$ is always positive semi-definite or negative semi-definite in this case. Since $\varphi_{j}(\lambda)$ and $\psi_{j}(\lambda)$ are linearly independent, we find that, if $f(\lambda)=2$, then $f^{\prime}(\lambda)=0$ if and only if $\delta_{j}(\lambda) \equiv 0$ for all $0 \leq j \leq N-1$. In this case, we find $I$ is a zero matrix. Hence, we have $\psi_{N-1}(\lambda)=0, \varphi_{N-1}(\lambda)-\varphi_{N}(\lambda)=0$, and $\psi_{N}(\lambda)-\psi_{N-1}(\lambda)-\varphi_{N-1}(\lambda)=0$. Combined with (10) and (15), we can obtain $\psi_{N}(\lambda)=\varphi_{N-1}(\lambda)=\varphi_{N}(\lambda)=1$ and $\psi_{N-1}(\lambda)=0$. This implies that $\varphi_{j}(\lambda)$ and $\psi_{j}(\lambda)$ are two solutions of (1) and (5), i.e., $f^{\prime}(\lambda)=0$ whenever $f(\lambda)=2$ if and only if $\lambda$ is not simple. Similarly, it can be showed that $f^{\prime}(\lambda)=0$ whenever $f(\lambda)=-2$ if and only if $\lambda$ is not simple.

Next, we investigate the Neumann eigenvalues of (1) and (8), and give a proof of Theorem 3. Denote by $\left\{v_{k}\right\}_{k=0}^{N-1}$ the set of Neumann eigenvalues. We have the following lemmas.

Lemma 4 For $0 \leq k \leq N-1, f\left(v_{k}\right) \geq 2$ if kis even, and $f\left(v_{k}\right) \leq-2$ if $k$ is odd.

Proof Let $\phi_{n}(\lambda) \equiv c_{1} \varphi_{n}(\lambda)+c_{2} \psi_{n}(\lambda)$. Then $\phi_{n}\left(v_{k}\right)$ is an eigenfunction of (1) and (8) if

$$
\phi_{-1}\left(v_{k}\right)-\phi_{0}\left(v_{k}\right)=0, \quad \phi_{N}\left(v_{k}\right)-\phi_{N-1}\left(v_{k}\right)=0,
$$

i.e.,

$$
\begin{aligned}
& c_{1} \varphi_{-1}\left(v_{k}\right)+c_{2} \psi_{-1}\left(v_{k}\right)=c_{1} \varphi_{0}\left(v_{k}\right)+c_{2} \psi_{0}\left(v_{k}\right), \\
& c_{1} \varphi_{N}\left(v_{k}\right)+c_{2} \psi_{N}\left(v_{k}\right)=c_{1} \varphi_{N-1}\left(v_{k}\right)+c_{2} \psi_{N-1}\left(v_{k}\right) .
\end{aligned}
$$

By (9), we have

$$
c_{2}=0, \quad c_{1}\left[\varphi_{N}\left(v_{i}\right)-\varphi_{N-1}\left(v_{i}\right)\right]=0 .
$$

Since $c_{1}$ and $c_{2}$ are not both zero, we find $\varphi_{N}\left(v_{k}\right)-\varphi_{N-1}\left(v_{k}\right)=0$. This implies that $\varphi_{n}\left(v_{k}\right)$ is an eigenfunction of (1) and (8) with respect to $v_{k}$.

On the other hand, by (10), we find

$$
\begin{aligned}
1 & =\varphi_{N-1}\left(v_{k}\right) \psi_{N}\left(v_{k}\right)-\varphi_{N}\left(v_{k}\right) \psi_{N-1}\left(v_{k}\right) \\
& =\varphi_{N-1}\left(v_{k}\right)\left[\psi_{N}\left(v_{k}\right)-\psi_{N-1}\left(v_{k}\right)\right]+\left[\varphi_{N-1}\left(v_{k}\right)-\varphi_{N}\left(v_{k}\right)\right] \psi_{N-1}\left(v_{k}\right) \\
& =\varphi_{N-1}\left(v_{k}\right)\left[\psi_{N}\left(v_{k}\right)-\psi_{N-1}\left(v_{k}\right)\right] .
\end{aligned}
$$

Hence,

$$
\psi_{N}\left(v_{k}\right)-\psi_{N-1}\left(v_{k}\right)=\frac{1}{\varphi_{N-1}\left(v_{k}\right)} .
$$

This implies that

$$
f\left(v_{k}\right) \equiv \varphi_{N-1}\left(v_{k}\right)+\psi_{N}\left(v_{k}\right)-\psi_{N-1}\left(v_{k}\right)=\varphi_{N-1}\left(v_{k}\right)+\frac{1}{\varphi_{N-1}\left(v_{k}\right)} .
$$


Finally, since $\varphi_{n}\left(v_{k}\right), n=0,1,2, \ldots, N-1$, changes sign $k$ times and $\varphi_{0}\left(v_{k}\right)=1$, we find $\operatorname{sgn}\left(\varphi_{N-1}\left(v_{k}\right)\right)=(-1)^{k}$. This implies that

$$
f\left(v_{k}\right)=\varphi_{N-1}\left(v_{k}\right)+\frac{1}{\varphi_{N-1}\left(v_{k}\right)} \begin{cases}\geq 2 & \text { if } k \text { is even, } \\ \leq-2 & \text { if } k \text { is odd }\end{cases}
$$

Lemma $5 f^{\prime}(\lambda)<0$ for all $\lambda<v_{0}$.

Proof Suppose $f(\lambda)= \pm 2$ for some $\lambda \neq v_{i}$, and $\delta_{j}(\lambda) \neq 0$. By (10) and (15), we calculate that

$$
\begin{aligned}
\delta_{j}(\lambda)= & {\left[\varphi_{N-1}(\lambda)-\varphi_{N}(\lambda)\right] \psi_{j}^{2}(\lambda)+\left[\psi_{N}(\lambda)-\psi_{N-1}(\lambda)-\varphi_{N-1}(\lambda)\right] \varphi_{j}(\lambda) \psi_{j}(\lambda) } \\
& +\psi_{N-1}(\lambda) \varphi_{j}^{2}(\lambda) \\
= & {\left[\varphi_{N-1}(\lambda)-\varphi_{N}(\lambda)\right]\left\{\psi_{j}(\lambda)+\frac{\left[\psi_{N}(\lambda)-\psi_{N-1}(\lambda)-\varphi_{N-1}(\lambda)\right]}{2\left[\varphi_{N-1}(\lambda)-\varphi_{N}(\lambda)\right]} \varphi_{j}(\lambda)\right\}^{2} } \\
& +\psi_{N-1}(\lambda) \varphi_{j}^{2}(\lambda)-\frac{\left[\psi_{N}(\lambda)-\psi_{N-1}(\lambda)-\varphi_{N-1}(\lambda)\right]^{2}}{4\left[\varphi_{N-1}(\lambda)-\varphi_{N}(\lambda)\right]} \varphi_{j}^{2}(\lambda) \\
= & {\left[\varphi_{N-1}(\lambda)-\varphi_{N}(\lambda)\right]\left\{\psi_{j}(\lambda)+\frac{\left[\psi_{N}(\lambda)-\psi_{N-1}(\lambda)-\varphi_{N-1}(\lambda)\right]}{2\left[\varphi_{N-1}(\lambda)-\varphi_{N}(\lambda)\right]} \varphi_{j}(\lambda)\right\}^{2} . }
\end{aligned}
$$

We find

$$
f^{\prime}(\lambda)=\left[\varphi_{N}(\lambda)-\varphi_{N-1}(\lambda)\right] \sum_{j=0}^{N-1}\left\{\psi_{j}(\lambda)+\frac{\psi_{N+1}(\lambda)-\psi_{N}(\lambda)-\varphi_{N}(\lambda)}{2\left[\varphi_{N}(\lambda)-\varphi_{N+1}(\lambda)\right]} \varphi_{j}(\lambda)\right\}^{2},
$$

and hence $f^{\prime}(\lambda)$ and $\varphi_{N}(\lambda)-\varphi_{N-1}(\lambda)$ have the same sign. Since $f(\lambda)$ is continuous on $\lambda$, and $\nu_{0}$ is the first Neumann eigenvalue and is simple, we find $f^{\prime}(\lambda)<0$ if $\lambda<\nu_{0}$.

By the above discussion and combining the result of [26, Theorem 3.1], we find that if $N$ is odd,

$$
v_{0} \leq \lambda_{0}<\tilde{\lambda}_{1} \leq{ }_{v_{1}}^{\mu_{1}} \leq \tilde{\lambda}_{2}<\lambda_{1} \leq{ }_{v_{2}}^{\mu_{2}} \leq \lambda_{2}<\cdots<\lambda_{N-2} \leq \begin{gathered}
\mu_{N-1} \\
v_{N-1}
\end{gathered} \leq \lambda_{N-1}<\tilde{\lambda}_{N}
$$

and if $N$ is even,

$$
v_{0} \leq \lambda_{0}<\tilde{\lambda}_{1} \leq{ }_{v_{1}}^{\mu_{1}} \leq \tilde{\lambda}_{2}<\lambda_{1} \leq \begin{aligned}
& \mu_{2} \\
& v_{2}
\end{aligned} \leq \lambda_{2}<\cdots<\tilde{\lambda}_{N-1} \leq \begin{aligned}
& \mu_{N-1} \\
& v_{N-1}
\end{aligned} \tilde{\lambda}_{N}<\lambda_{N-1} .
$$

\section{The order relation of the first Dirichlet eigenvalue and the second Neumann eigenvalue}

In this section, we investigate the order relation between the first Dirichlet eigenvalue and the second Neumann eigenvalue. Denote by $\left(v_{k}, \vec{w}_{k}\right)_{k=0}^{N-1}$ the Neumann eigenpairs of (1) and (8) with $\left\|\vec{w}_{k}\right\|_{2}=1$. In particular, $\vec{w}_{k}$ can be chosen to have exactly $k$ sign changes. We have the following lemma.

Lemma 6 If $\max _{k \in[0, N-1]} q_{k} \leq v_{1}$, then $\vec{w}_{1}$ is decreasing. 
Proof Denote $\vec{w}_{1}=\left(w_{1,0}, w_{1,1}, w_{1,2}, \ldots, w_{1, N-1}\right)$. Since $\vec{w}_{1}$ satisfies the Neumann boundary conditions, we also assume $w_{1,-1}=w_{1,0}$ and $w_{1, N}=w_{1, N-1}$. Then we have

$$
\begin{aligned}
& w_{1, j+1}=\left(2+q_{j}-v_{1}\right) w_{1, j}-w_{1, j-1} \quad \text { for } j=0,1,2, \ldots, N-1, \\
& w_{1,-1}=w_{1,0}, \quad w_{1, N}=w_{1, N-1} .
\end{aligned}
$$

First, we evaluate that

$$
w_{1,1}=\left(2+q_{0}-v_{1}\right) w_{1,0}-w_{1,-1}=w_{1,0}+\left(q_{0}-v_{1}\right) w_{1,0} \leq w_{1,0} .
$$

Now, assume $w_{1, j} \leq w_{1, j-1}$. Then

$$
w_{1, j+1}=\left(2+q_{j}-v_{1}\right) w_{1, j}-w_{1, j-1}=w_{1, j}+\left(q_{j}-v_{1}\right) w_{1, j}+w_{1, j}-w_{1, j-1} \leq w_{1, j} .
$$

By induction, we find $\vec{w}_{1}$ is decreasing.

Proof of Theorem 2 Define $\vec{z}=\left(z_{j}\right)_{j=0}^{N}$ where $z_{j}=w_{1, j}-w_{1, j-1}$ for $j=0,1,2, \ldots, N$. Then $z_{0}=$ $z_{N}=0$. In particular, by Lemma $6, z_{j}>0$. Hence, $\vec{z}$ is the first Dirichlet eigenfunction. By the variational principle, we have

$$
\mu_{1} \leq \frac{\langle\vec{z}, H \vec{z}\rangle}{\langle\vec{z}, \vec{z}\rangle}
$$

where $(H z)_{j}=-z_{j+1}+\left(2+q_{j}\right) z_{j}-z_{j-1}$. Since $\vec{w}_{1}$ changes sign only once and $q_{k}$ is symmetric, we find that if $N-1$ is even, then $w_{1, j}>0$ for $j \in\left[0, \frac{N-1}{2}\right), w_{1, j}<0$ for $j \in\left(\frac{N-1}{2}, N-1\right]$, and $w_{1, \frac{N-1}{2}}=0$; while if $N-1$ is odd, then $w_{1, j}>0$ for $j \in\left[0, \frac{N-2}{2}\right]$ and $w_{1, j}<0$ for $j \in$ $\left[\frac{N}{2}, N-1\right]$. Furthermore, since $q_{k}$ is symmetric and symmetric decreasing, we have, for $j=0,1, \ldots, N-1$,

$$
\begin{aligned}
- & z_{j+1}+\left(2+q_{j}\right) z_{j}-z_{j-1} \\
& =-\left(w_{1, j+1}-w_{1, j}\right)+\left(2+q_{j}\right)\left(w_{1, j}-w_{1, j-1}\right)-\left(w_{1, j-1}-w_{1, j}\right) \\
& =\left(-w_{1, j+1}+\left(2+q_{j}\right) w_{1, j}-w_{1, j-1}\right)-\left(-w_{1, j}+\left(2+q_{j}\right) w_{1, j-1}-w_{1, j-1}\right) \\
& \leq\left(-w_{1, j+1}+\left(2+q_{j}\right) w_{1, j}-w_{1, j-1}\right)-\left(-w_{1, j}+\left(2+q_{j-1}\right) w_{1, j-1}-w_{1, j-1}\right) \\
& =v_{1} w_{1, j}-v_{1} w_{1, j-1} \\
& =v_{1} z_{j} .
\end{aligned}
$$

This implies that

$$
\mu_{1} \leq \frac{\langle\vec{z}, H \vec{z}\rangle}{\langle\vec{z}, \vec{z}\rangle}=\frac{\nu_{1} \sum_{i=0}^{N-1} z_{i}^{2}}{\sum_{i=0}^{N-1} z_{i}^{2}}=v_{1}
$$

The equality holds if and only if $q_{j}=q_{0}$ for $j=1,2, \ldots, N-1$. 


\section{The lower bound of the first Neumann eigenvalue gap}

In this section, we give an optimal lower bound of the first Neumann eigenvalue gap.

Lemma 7 Consider (1) and (8), and let $q_{n}(t)$ be a one-parameter family of potential sequences such that $q_{n}^{\prime}(t)$ exists. Then

$$
v_{k}^{\prime}(t)=\sum_{j=0}^{N-1} q_{j}^{\prime}(t) w_{k, j}^{2}(t) .
$$

Proof Consider (1) and (8) with the potential sequence $q_{j}(t)$ :

$$
w_{k, j+1}(t)=\left(2+q_{j}(t)-v_{k}(t)\right) w_{k, j}(t)-w_{k, j-1}(t)
$$

Differentiating the above equation with respect to $t$, we have

$$
w_{k, j+1}^{\prime}(t)=\left(q_{j}^{\prime}(t)-v_{k}^{\prime}(t)\right) w_{k, j}(t)+\left(2+q_{j}(t)-v_{k}(t)\right) w_{k, j}^{\prime}(t)-w_{k, j-1}^{\prime}(t)
$$

Furthermore, we can obtain

$$
\begin{aligned}
& w_{k, j+1}^{\prime}(t) w_{k, j}(t)-w_{k, j+1}(t) w_{k, j}^{\prime}(t) \\
& \quad=\left(q_{j}^{\prime}(t)-v_{k}^{\prime}(t)\right) w_{k, j}^{2}(t)+w_{k, j-1}(t) w_{k, j}^{\prime}(t)-w_{k, j}(t) w_{k, j-1}^{\prime}(t) .
\end{aligned}
$$

Summing up the above equation from $j=0$ to $j=N-1$, we have

$$
v_{k}^{\prime}(t) \sum_{j=0}^{N-1} w_{k, j}^{2}(t)=\sum_{j=0}^{N-1} q_{j}^{\prime}(t) w_{k, j}^{2}(t) .
$$

Since $\left\|\vec{w}_{k}\right\|_{2}=1$, we find

$$
v_{k}^{\prime}(t)=\sum_{j=0}^{N-1} q_{j}^{\prime}(t) w_{k, j}^{2}(t)
$$

Lemma 8 There exist $j_{1}, j_{2} \in(0, N-1)$ with $j_{1}<j_{2}$ such that

$$
w_{1, j}^{2}(t)-w_{0, j}^{2}(t) \begin{cases}\geq 0 & \text { if } j \in\left[0, j_{1}\right] \cup\left[j_{2}, N-1\right] \\ \leq 0 & \text { if } j \in\left[j_{1}, j_{2}\right] .\end{cases}
$$

Proof First, since $\vec{w}_{1}$ changes sign only once, we may assume $w_{1, j} \geq 0$ for $j \in[0, \bar{k}]$ and $w_{1, j} \leq 0$ for $j \in[\bar{k}, N-1]$. Since

$$
\begin{aligned}
& w_{0,1}=\left(2+q_{0}-v_{0}\right) w_{0,0}-w_{0,-1}=\left(1+q_{0}-v_{0}\right) w_{0,0}, \\
& w_{1,1}=\left(2+q_{0}-v_{1}\right) w_{1,0}-w_{1,-1}=\left(1+q_{0}-v_{1}\right) w_{1,0},
\end{aligned}
$$

we have

$$
w_{0,1} w_{1,0}-w_{0,0} w_{1,1}=\left(v_{1}-v_{0}\right) w_{0,0} w_{1,0}>0,
$$


and hence

$$
\frac{w_{1,0}}{w_{0,0}}>\frac{w_{1,1}}{w_{0,1}}
$$

Now, for $j \in[0, \bar{k}-1]$, assume $\frac{w_{1, j}}{w_{0, j}}>\frac{w_{1, j+1}}{w_{0, j+1}}$ holds. Then

$$
w_{0, j+2} w_{1, j+1}-w_{0, j+1} w_{1, j+2}=\left(v_{1}-v_{0}\right) w_{0, j+1} w_{1, j+1}+w_{0, j+1} w_{1, j}-w_{0, j} w_{1, j+1}>0,
$$

and hence

$$
\frac{w_{1, j+1}}{w_{0, j+1}}>\frac{w_{1, j+2}}{w_{0, j+2}}
$$

By induction, we find

$$
\frac{w_{1,0}}{w_{0,0}}>\frac{w_{1,1}}{w_{0,1}}>\cdots>\frac{w_{1, \bar{k}-1}}{w_{0, \bar{k}-1}}>\frac{w_{1, \bar{k}}}{w_{0, \bar{k}}}
$$

This implies that there exists $j_{1} \in(0, \bar{k})$ such that

$$
\frac{w_{1, j_{1}}}{w_{0, j_{1}}} \geq 1>\frac{w_{1, j_{1}+1}}{w_{0, j_{1}+1}}
$$

Hence,

$$
w_{1, j}^{2}(t)-w_{0, j}^{2}(t) \begin{cases}\geq 0 & \text { if } j \in\left[0, j_{1}\right] \\ \leq 0 & \text { if } j \in\left[j_{1}, \bar{k}\right]\end{cases}
$$

Similarly, it can be showed that there exists $j_{2} \in(\bar{k}, N-1)$ such that

$$
w_{1, j}^{2}(t)-w_{0, j}^{2}(t) \begin{cases}\geq 0 & \text { if } j \in\left[j_{2}, N-1\right] \\ \leq 0 & \text { if } j \in\left[\bar{k}, j_{2}\right] .\end{cases}
$$

The proof is complete.

Now, we are prepared to prove Theorem 3.

\section{Proof of Theorem 3}

First, according to Lemma 8 and since $q_{j}$ is symmetric, we find that there exists $\bar{j} \in(0, N-$ 1) such that

$$
w_{1, j}^{2}(t)-w_{0, j}^{2}(t) \begin{cases}\geq 0 & \text { if } j \in[0, \bar{j}] \cup[N-1-\bar{j}, N-1] \\ \leq 0 & \text { if } j \in\left[\bar{j}, j_{2}\right] .\end{cases}
$$

Now, consider

$$
S=\left\{q_{n}: q_{n} \text { is symmetric and symmetric increasing }\right\} .
$$


Then $v_{1}-v_{0}$ attains its minimum at some $\hat{q}_{n} \in S$. Let $q_{j}(t)=(1-t) \hat{q}_{j}+t \hat{q}_{\bar{j}}$. By Lemma 7 , we find

$$
v_{1}^{\prime}(t)-v_{0}^{\prime}(t)=\sum_{j=0}^{N-1} q_{j}^{\prime}(t)\left(w_{1, j}^{2}(t)-w_{0, j}^{2}(t)\right)=\sum_{j=0}^{N-1}\left(\hat{q}_{j}-\hat{q}_{j}\right)\left(w_{1, j}^{2}(t)-w_{0, j}^{2}(t)\right)<0 .
$$

This implies that

$$
v_{1}(t)-v_{0}(t)>v_{1}(1)-v_{0}(1)=\left(v_{1}-v_{0}\right)\left[\hat{q}_{j}\right]=2\left[1-\cos \left(\frac{\pi}{N+1}\right)\right] .
$$

\section{Acknowledgements}

The author was partially supported by the Ministry of Science and Technology of the Republic of China under the grant number MOST 106-2115-M-152-001.

\section{Funding}

Not applicable.

\section{Availability of data and materials}

Not applicable.

\section{Competing interests}

The author declares that they have no competing interests.

\section{Authors' contributions}

All authors read and approved the final manuscript.

\section{Publisher's Note}

Springer Nature remains neutral with regard to jurisdictional claims in published maps and institutional affiliations.

\section{Received: 27 August 2019 Accepted: 18 October 2020 Published online: 23 October 2020}

\section{References}

1. Ashbaugh, M.S., Benguria, R.D.: Optimal lower bound for the gap between the first two eigenvalues of one-dimensional Schrodinger operators with symmetric single-well potentials. Proc. Am. Math. Soc. 105, 419-424 (1989)

2. Ashbaugh, M.S., Benguria, R.D.: Some eigenvalue inequalities for a class of Jacobi matrices. Linear Algebra Appl. 136, 215-234 (1990)

3. Atkinson, F.V.: Discrete and Continuous Boundary Problems. Academic Press, New York (1964)

4. Birkhoff, G., Rota, G.C.: Ordinary Differential Equations. Wiley, New York (1989)

5. Borg, G.: Eine Umkehrung der Sturm-Liouvilleschen Eigenwertaufgabe. Bestimmung der Differentialgleichung durch die Eigenwerte. Acta Math. 78, 1-96 (1946)

6. Cheng, Y.H.: Eigenvalue problems with p-Laplacian operators. Electron. J. Differ. Equ. 2014, 139 (2014)

7. Cheng, Y.H., Kung, S.Y., Law, C.K., Lian, W.C.: The dual eigenvalue problems for the Sturm-Liouville system. Comput. Math. Appl. 60, 2556-2563 (2010)

8. Chern, H.H., Shen, C.L.: On the maximum and minimum of some functionals for the eigenvalue problem of Sturm-Liouville type. J. Differ. Equ. 107, 68-79 (1994)

9. Coddington, E.A., Levinson, N.: Theory of Ordinary Differential Equations. McGraw-Hill, New York (1955)

10. Hald, H.: Inverse eigenvalue problems for Jacobi matrices. Linear Algebra Appl. 14, 63-85 (1976)

11. Hochstadt, H.: On the determination of a Hill's equation from its spectrum. Arch. Ration. Mech. Anal. 19, 353-362 (1965)

12. Hochstadt, H.: On some inverse problems in matrix theory. Arch. Math. 18, 201-207 (1967)

13. Hochstadt, H.: On the construction of a Jacobi matrix from spectral data. Linear Algebra Appl. 8, 435-446 (1974)

14. Hochstadt, H.: A generalization of Borg's inverse theorem for Hill's equations. J. Math. Anal. Appl. 102, $599-605$ (1984)

15. Horvath, M.: On the first two eigenvalues of Sturm-Liouville operators. Proc. Am. Math. Soc. 131, 1215-1224 (2003)

16. Huang, M.J.: The first instability interval for Hill equations with symmetric single well potentials. Proc. Am. Math. Soc. $125,775-778(1997)$

17. Ji, J., Yang, B.: Eigenvalue comparisons for second order difference equations with Neumann boundary conditions. Linear Algebra Appl. 425, 171-183 (2007)

18. Jirari, A.: Second-order Sturm-Liouville difference equations and orthogonal polynomials. Mem. Am. Math. Soc. 113, $1-138(1995)$

19. Keller, J.B. The minimum ratio of two eigenvalues. SIAM J. Appl. Math. 31, 485-491 (1976)

20. Lavine, R.: The eigenvalue gap for one-dimensional convex potentials. Proc. Am. Math. Soc. 121(3), $815-821$ (1994)

21. Levitan, B.M., Sargsjan, I.S.: Sturm-Liouville and Dirac Operators. Kluwer Academic, Dordrecht (1991)

22. Magnus, W., Winkler, S.: Hill's Equations. Wiley, New York (1966) 
23. Poschel, I., Trubowitz, E.: Inverse Spectral Theory. Academic Press, New York (1987)

24. Shi, Y., Chen, S.: Spectral theory of second-order vector difference equations. J. Math. Anal. Appl. 239, 195-212 (1999)

25. Shieh, C.T.: Some inverse problems on Jacobi matrices. Inverse Probl. 20, 589-600 (2004)

26. Wang, Y., Shi, Y.: Eigenvalues of second-order difference equations with periodic and antiperiodic boundary conditions. J. Math. Anal. Appl. 309, 56-69 (2005)

Submit your manuscript to a SpringerOpen ${ }^{\odot}$ journal and benefit from:

- Convenient online submission

$\checkmark$ Rigorous peer review

- Open access: articles freely available online

- High visibility within the field

- Retaining the copyright to your article

Submit your next manuscript at $\gg$ springeropen.com 